\title{
Effect of mindfulness on physical activity in primary healthcare patients: a randomised controlled trial pilot study
}

\author{
Peter Nymberg ${ }^{1,2^{*}}$ (D), Susanna Calling ${ }^{1,2}$, Emelie Stenman $^{1,2}$, Karolina Palmér $^{1}$, Eva Ekvall Hansson ${ }^{3}$,
} Kristina Sundquist ${ }^{1,2}$, Jan Sundquist ${ }^{1,2}$ and Bengt Zöller ${ }^{1,2}$

\begin{abstract}
Increased physical activity can have health benefits among inactive individuals. In Sweden, the healthcare system uses physical activity on prescription (PAP) to motivate patients to increase their physical activity level. Mindfulness may further heighten the internal motivation to engage in physical activity. However, previous research has not demonstrated clear evidence of such an association.

Aim: Examine the feasibility of the study design as a preparation for a full-scale study, and examine the differences, between three interventions, in change over time in physical activity levels and in related variables.

Method: Comparison between three different interventions in an ordinary primary health care setting: PAP, mindfulness, and a combination of PAP and mindfulness. Physical activity was measured with self-report and ACTi Graph GT1X activity monitor. Statistical analysis was performed with a mixed-effect model to account for repeated observations and estimate differences both within groups and between groups at 3- and 6-months follow-up.
\end{abstract}

Results: Between September 2016 and December 2018, a total of 88 participants were randomised into three groups. The total dropout rate was $20.4 \%$, the attendance rate to the mindfulness courses (52\%> 6 times) and the web-based mindfulness training ( $8 \%>800 \mathrm{~min})$ was low according to the stated feasibility criteria. Eleven participants were excluded from analysis due to low activity monitor wear time. Neither the activity monitor data nor self-reported physical activity showed any significant differences between the groups.

Conclusion: The study design needs adjustment for the mindfulness intervention design before a fully scaled study can be conducted. A combination of PAP and mindfulness may increase physical activity and self-rated health more than PAP or mindfulness alone.

Trial registration: ClinicalTrials.gov, registration number NCT02869854. Regional Ethical Review Board in Lund registration number 2016/404.

\section{Key messages regarding feasibility}

- What uncertainties existed regarding the feasibility? An association between being mindful and physical activity level has been suggested. However, it is still unknown whether it is mindfulness that leads to

\footnotetext{
* Correspondence: peter.nymberg@med.lu.se

${ }^{1}$ Center for Primary Health Care Research, Region Skåne, Lund, Sweden ${ }^{2}$ Department of Clinical Sciences, Malmö Lund University, Lund, Sweden Full list of author information is available at the end of the article
}

increased physical activity or vice versa. Physical activity on prescription (PAP) is an established method for promoting physical activity and we wanted to examine the feasibility of comparing sedentary primary care patients that were randomized into PAP, mindfulness training or a combination of both. Feasibility was estimated by measuring adherence, recruitment rate and number of dropouts. The results will be used to design a

(C) The Author(s). 2021 Open Access This article is licensed under a Creative Commons Attribution 4.0 International License, which permits use, sharing, adaptation, distribution and reproduction in any medium or format, as long as you give appropriate credit to the original author(s) and the source, provide a link to the Creative Commons licence, and indicate if changes were made. The images or other third party material in this article are included in the article's Creative Commons licence, unless indicated otherwise in a credit line to the material. If material is not included in the article's Creative Commons licence and your intended use is not permitted by statutory regulation or exceeds the permitted use, you will need to obtain permission directly from the copyright holder. To view a copy of this licence, visit http://creativecommons.org/licenses/by/4.0/ The Creative Commons Public Domain Dedication waiver (http://creativecommons.org/publicdomain/zero/1.0/) applies to the data made available in this article, unless otherwise stated in a credit line to the data. 
larger scale randomized controlled study with a proper power calculation.

- What are the key findings?

Of 136 eligible patients, a total of 88 volunteered to participate thus resulting in a recruitment rate of $64.7 \%$. Only $52 \%$ of the patients, which were randomized to only mindfulness or the combination group, attended the mindfulness course, and only $8 \%$ did 800 min or more of the associated webbased training. Besides the low dropout rate of $20.4 \%$, there was loss to follow-up regarding accelerometer data. There was a tendency towards an increased effect of PAP on physical activity level when mindfulness training was added.

- What are the implications of the feasibility findings for design of the main study?

The mindfulness intervention needs to be remoulded to improve compliance in a larger-scale study. The type of accelerometers used (hip/wrist worn) also need to be considered to minimize the loss of objective physical activity data to follow-up.

\section{Introduction}

A lifestyle with an adequate amount of physical activity can decrease the risk of both somatic and mental illness [1-4]. Although people in northern Europe are generally physically active [5], they report more sedentary time than their southern European counterparts [6]. A study regarding sedentary behaviour, among 50- to 64-yearold Swedish adults, showed that only $7.1 \%$ of the 948 participants fulfilled the World Health Organization's (WHO) recommendation for physical activity [7]. Making individuals change their physical activity behaviour is an ongoing challenge. A systematic review estimated that 12 sedentary adults need to be treated with a physical activity promotion intervention in order to make one of them achieve the recommended physical activity level at one year follow-up [8]. In Sweden, the health care services recommend the use of physical activity on prescription (PAP) as a complementary treatment to motivate patients to increase their physical activity level; the treatment addresses both primary and secondary prevention of illness. The written prescription in the Swedish PAP model can be a proposal for an activity or an extensive solution with a supportive structure depending on the patient's needs and level of motivation. The Swedish PAP model has been associated with up to $60 \%$ increased activity levels, but the effect has not shown sustainability over time [9-11]. In some of the studies complemented with pedometers, the most common way to report the effect of PAP has been by selfreported measures [11]. To the authors' knowledge, only one study has used activity monitors to measure the effect of the Swedish PAP model [12] and it failed to show significantly increased levels of moderate to vigorous physical activity (MVPA).

Research has demonstrated that satisfaction can play a crucial role in changing a behaviour such as physical activity [13], smoking cessation [14] or weight loss [15]. It has been suggested that satisfaction can be increased both by the awareness in a specific positive situation and by the reduction of negative thoughts, e.g. about physical activity [16]; something which may be facilitated by practising mindfulness [17]. All people have a varying intrinsic, albeit modifiable, trait to be aware of the present moment-dispositional mindfulness [18]. Mindfulness can be exerted as sitting meditation but also as an approach to everyday life [19]. The practice of mindfulness might give the individual an orientation toward one's experiences in the present moment [20]. Being mindful in a specific situation and, in addition, satisfaction are suggested to be consecutive mediators for the path between possessing a dispositional tendency to be mindful and physical activity [17]. This might explain why self-reported mindfulness seems to mediate the relationship between intrinsic motivation and the physical activity level [21]. In addition, mindfulness has been shown to increase pain tolerance [22, 23]. In other words, practising mindfulness can make it easier to experience satisfaction and mitigate discomfort connected with physical activity and, in this way, support the change from a physically inactive to a physically active behaviour. Recent research has shown a lower decrease-if mindfulness was being practised-in physical activity due to seasonal decline compared with a control group [24]. A review, which was conducted to investigate the role of mindfulness in physical behaviour changes, revealed that mindfulness interventions influenced the physical activity outcomes in a positive direction in a majority of the 40 included studies [25]. Mindfulnessbased interventions were suggested to be successful if they targeted psychological factors related to increased physical activity, such as self-efficacy and acceptance. Even if mindfulness correlates with factors that can influence the increase of physical activity, it has been shown that regular exercise can lead to an increased dispositional mindfulness [26]. Thus, mindfulness may have an important role to play concerning motivation by reinforcing satisfaction with physical activity [25].

\section{Aim}

To examine the feasibility of the study design as a preparation for a full-scale study.

The intervention outcome was differences of change in physical activity level over time between three groups: PAP, mindfulness and a combination containing both 
PAP and mindfulness, in a population with insufficient self-reported levels of physical activity.

\section{Methods/design Participants}

This was a pilot-study preparing for a larger-scale randomised trial. For detailed information about the study, we refer to the published study-protocol [27]. Men and women, which could speak fluent Swedish and were aged between 40 and 65 years, visiting their primary healthcare clinic for any reason were asked to rate their physical activity level. Those with a self-rated physical activity level below the WHO recommendations were asked to participate in the study. We excluded from the study (within 6 weeks before study entry) those with dementia, severe mental disorder, unstable untreated angina pectoris or myocardial infarction. The criteria of physical activity were defined according to the WHO guidelines, in which the lower limits for sufficient activity are set to $150 \mathrm{~min}$ per week of moderate intensity or 75 min per week of high intensity exercise.

\section{Setting}

The pilot study involved three primary health care centres, recruited on voluntary basis, in the county of Scania in southern Sweden. In total, there are approximately 164 primary health care centres in the county. Scania has approximately 1.2 million residents and about 400 , 000 of these are aged between 40 and 65 years old.

\section{Outcome measures}

\section{Feasibility criteria for a successful design}

1. Recruitment rate more than $30 \%$ [28].

2. Dropout rate of less than $30 \%$.

3. Compliance to the mindfulness course: $\geq 70 \%$ of those randomised to any of the groups containing mindfulness should participate in at least $75 \%$ of the mindfulness meetings [29].

4. Web-based practice for at least 20 min 5 days per week ( $800 \mathrm{~min}$ ) by $70 \%$ of patients randomised to any group containing mindfulness [30].

\section{Intervention outcome}

All measurements and questionnaires were collected at baseline, after 3 months and after 6 months.

The primary intervention outcome was changed level of physical activity, self-reported and measured by ACTi Graph GT1X activity monitors. We used the same definitions and methods to handle the activity monitor data as in previously published research regarding physical activity $[7,12]$. Activity monitor data were divided into sedentary, light physical activity (LIPA) and moderate to vigorous physical activity (MVPA). The participants were instructed to wear the activity monitor every day for a week before randomisation, at 3 months and at 6 months follow-up. Wear time was defined by subtracting non-wear time from $24 \mathrm{~h}$. Non-wear time was defined as at least 60 consecutive minutes with no movement $(0$ counts per minute), with allowance for maximum $2 \mathrm{~min}$ of counts between 0 and 100 [7,31]. We considered $\geq$ $600 \mathrm{~min}$ wear time per day for at least 4 days to be valid compliance $[7,12]$. Due to a small sample size, we did not demand four consecutive days with valid wear time, and we did not differ between weekdays and weekends. Average was expressed as total counts divided by wear time in minutes per day (counts per minute) and averaged over worn days. Registrations below 100 counts per minute were determined as being sedentary $[7,12,32]$. 100-2019 counts per minute were considered as LIPA and $>2020$ counts per minute as MVPA $[7,12]$. The results are presented as percentage sedentary, LIPA or MVPA per valid day and averaged over the number of valid days [12] self-reported daily activity (e.g. gardening, slow walks, biking) was measured by an eight-step scale ( 0 = $0 \mathrm{~min} /$ week, $7 \geq 300 \mathrm{~min} /$ week), and self-reported leisure time activity (e.g. running, football) was measured by a seven-step scale $(0=0 \mathrm{~min} /$ week, $6 \geq 200 \mathrm{~min} /$ week $)$.

\section{Secondary intervention outcomes}

1. Change in self-rated health (SRH) between baseline and follow-up, measured with a five-step scale (15): very poor, poor, fair, good or very good.

2. Change in blood pressure, weight or serum lipids between baseline and follow-up.

3. Change in insomnia problems as measured with insomnia severity index (ISI) [33] between baseline and follow-up.

4. Change in mindfulness measured with five facets of mindfulness questionnaire (FFMQ) [34] between baseline and follow-up.

\section{Interventions}

Participants in the PAP group were prescribed Swedish PAP $[9,35]$, which is the recommended treatment for physically inactive patients and adjusted to each patient's individual preferences. The participants in the mindfulness group received a two-hour long mindfulness group session once a week for 8 weeks and were instructed to practise mindfulness for 20 min every day. The mindfulness course [29] was based on both Mindfulness-Based Stress Reduction (MBSR) and Mindfulness-Based Cognitive Therapy (MBCT) and included meditative exercises. The patients received instructions concerning the daily mindfulness practice with meditative exercises via a web-based program [29]. The instructions included breathing technique and body scan. The combination 
Table 1 Patient characteristics for all participants at baseline, 3 months and 6 months

\begin{tabular}{|c|c|c|c|}
\hline Randomisation group & PAP & Mindfulness & Combination \\
\hline \multicolumn{4}{|l|}{ Baseline $n(\%)$} \\
\hline Women & $22(76)$ & $22(73)$ & $20(69)$ \\
\hline Men & $7(24)$ & $8(27)$ & $9(31)$ \\
\hline \multicolumn{4}{|l|}{3 months } \\
\hline Women & $18(72)$ & $19(79)$ & $17(65)$ \\
\hline Men & $4(18)$ & $5(21)$ & $9(35)$ \\
\hline \multicolumn{4}{|l|}{6 months } \\
\hline Women & $17(77)$ & $19(79)$ & $15(63)$ \\
\hline Men & $5(23)$ & $5(21)$ & $9(37)$ \\
\hline \multicolumn{4}{|l|}{ Age median (range) } \\
\hline Baseline & $54(42-65)$ & $53(41-65)$ & $54(43-64)$ \\
\hline \multicolumn{4}{|c|}{ Percent of time in sedentary time ${ }^{a}$ mean ( $S D$, range) } \\
\hline Baseline & $66.2(6.7,78.2-50.2)$ & $65.5(9.2,80.3-43.7)$ & $66.7(8.8,80.0-47.2)$ \\
\hline 3 months follow-up & $66.7(6.7,78.3-56.3)$ & $65.5(7.5,78.9-51.3)$ & $67.5(8.1,81.2-51.3)$ \\
\hline 6 months follow-up & $65.0(6.8,73.6-50.0)$ & $65.9(9.4,81.0-36.2)$ & $64.0(9.1,78.4-45.6)$ \\
\hline \multicolumn{4}{|l|}{$\begin{array}{l}\text { Percent of time in LIPA } \\
\text { mean (SD, range) }\end{array}$} \\
\hline Baseline & $30.9(6.2,45.5-20.9)$ & $31.7(8.2,50.0-19.0)$ & $30.3(8.0,49.2-18.8)$ \\
\hline 3 months follow-up & $31.0(6.0,42.6-21.5)$ & $31.8(6.9,47.8-19.9)$ & $28.6(7.1,44.5-16.3)$ \\
\hline 6 months follow-up & $32.0(5.8,43.8-23.1)$ & $31.6(9.0,58.4-17.0)$ & $32.8(8.9,50.7-18.6)$ \\
\hline \multicolumn{4}{|c|}{ Percent of time in MVPA $^{\mathrm{a}}$ median (SD, range) } \\
\hline Baseline & $2.6(0.18,6.3-0.5)$ & $2.3(0.02,9.1-0.1)$ & $2.4(0.02,9.0-0.2)$ \\
\hline 3 months follow-up & $2.4(0.14,5.0-0.2)$ & 1.9. $(0.02,12.7-0.3)$ & $3.4(0.03,11.1-0.1)$ \\
\hline 6 months follow-up & $2.6(0.02,6.2-0.4)$ & $1.9(0.02,9.0-0.2)$ & $2.7(0.02,9.2-0.3)$ \\
\hline \multicolumn{4}{|c|}{ Weight kg median (range) } \\
\hline Baseline & $92.0(67-121)$ & $86.8(57-132)$ & $81.5(62-146)$ \\
\hline 3 months follow-up & $87.9(67-111)$ & $81.2(56-135)$ & $83(62-143)$ \\
\hline 6 months follow-up & $91.3(59-110)$ & $83.0(60-139)$ & $80.2(60-145)$ \\
\hline \multicolumn{4}{|c|}{ BMI $\mathbf{k g} / \mathbf{m}^{2}$ median (range) } \\
\hline Baseline & $31.4(21-43)$ & $29.9(22-44)$ & $28.5(21-40)$ \\
\hline 3 months follow-up & $30.0(21-42)$ & $28.6(22-43)$ & $28.2(21-39)$ \\
\hline 6 months follow-up & $29.4(21-42)$ & $28.6(23-44)$ & $27.9(23-39)$ \\
\hline \multicolumn{4}{|c|}{ Total cholesterol mmol/L mean (SD, range) } \\
\hline Baseline & $5.20(1.05,7.7-2.9)$ & $5.63(0.87,7.3-4.1)$ & $5.41(1.04,7.4-3.4)$ \\
\hline 3 months follow-up & $4.99(1.02,6.9-3.3)$ & $5.35(0.96,7.1-3.8)$ & $5.12(1.00,7-3.1)$ \\
\hline 6 months follow-up & $5.08(1.23,7.1-3)$ & $5.72(1.09,7.7-3.6)$ & $5.08(1.19,7.6-2.9)$ \\
\hline \multicolumn{4}{|c|}{ Low-density cholesterol mmol/L mean (SD, range) } \\
\hline Baseline & $3.43(1.04,5.9-1.3)$ & $3.80(0.92,5.7-2)$ & $3.66(0.94,5.5-2)$ \\
\hline 3 months follow-up & $3.15(0.97,5.1-1.7)$ & $3.51(1.02,5.8-2)$ & $3.52(1.01,5.3-1.7)$ \\
\hline 6 months follow-up & $3.26(1.14,5.3-1.5)$ & $3.83(1.04,5.9-1.9)$ & $3.57(1.15,6.3-1.6)$ \\
\hline \multicolumn{4}{|c|}{$\begin{array}{l}\text { High-density cholesterol } \\
\text { mmol/L median (SD, range) }\end{array}$} \\
\hline Baseline & $1.5(0.54,3.1-0.9)$ & $1.4(0.52,2.9-0.5)$ & $1.5(0.36,2.4-1.0)$ \\
\hline 3 months follow-up & $1.5(0.56,2.7-0.9)$ & $1.5(0.60,2.8-0.4)$ & $1.5(0.32,2.1-0.9)$ \\
\hline 6 months follow-up & $1.5(0.55,2.7-0.9)$ & $1.4(0.64,2.9-0.4)$ & $1.4(0.27,2.3-0.9)$ \\
\hline
\end{tabular}


Table 1 Patient characteristics for all participants at baseline, 3 months and 6 months (Continued)

\begin{tabular}{|c|c|c|c|}
\hline Randomisation group & PAP & Mindfulness & Combination \\
\hline \multicolumn{4}{|c|}{ Triglycerides $\mathbf{m m o l} / \mathbf{L}$ median (SD, range) } \\
\hline Baseline & $1.2(0.97,5.1-0.4)$ & $1.6(1.14,5.2-0.5)$ & $1.4(0.70,3.2-0.7)$ \\
\hline 3 months follow-up & $1.25(0.68,3-0.5)$ & $1.4(1.13,4.9-0.8)$ & $1.5(0.62,3.2-0.7)$ \\
\hline 6 months follow-up & $1.3(0.87,4.1-0.4)$ & $1.65(1.18,5.8-0.5)$ & $1.4(0.60,3.2-0.7)$ \\
\hline \multicolumn{4}{|l|}{$\begin{array}{l}\text { Systolic blood pressure } \\
\text { mmHg median (range) }\end{array}$} \\
\hline Baseline & $130(110-160)$ & $120(80-160)$ & $130(100-155)$ \\
\hline 3 months follow-up & $127(100-150)$ & $122(104-165)$ & $120(106-145)$ \\
\hline 6 months follow-up & $126(90-160)$ & $129(102-150)$ & $131(108-160)$ \\
\hline \multicolumn{4}{|c|}{$\begin{array}{l}\text { Diastolic blood pressure } \\
\mathbf{m m H g} \text { median (range) }\end{array}$} \\
\hline Baseline & $80(64-100)$ & $80(60-100)$ & $80(60-90)$ \\
\hline 3 months follow-up & $80(60-90)$ & $80(68-99)$ & $78(60-90)$ \\
\hline 6 months follow-up & $80(60-100)$ & $80(60-100)$ & $80(60-100)$ \\
\hline \multicolumn{4}{|c|}{ Leisure-time activity ${ }^{\mathrm{b}}(\min =0, \max =6)$ median (range) } \\
\hline Baseline & $1(1-5)$ & $2(1-3)$ & $2(1-4)$ \\
\hline 3 months follow-up & $3(1-6)$ & $2(1-3)$ & $3(1-6)$ \\
\hline 6 months follow-up & $3(1-6)$ & $2(1-6)$ & $3(1-6)$ \\
\hline \multicolumn{4}{|c|}{ 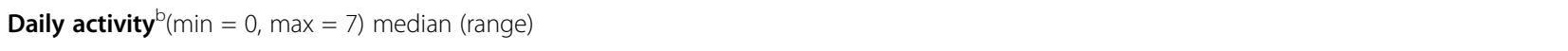 } \\
\hline Baseline & $3(1-7)$ & $3(1-6)$ & $4(1-5)$ \\
\hline 3 months follow-up & $5(2-7)$ & $3(1-7)$ & $5(1-6)$ \\
\hline 6 months follow-up & $5(3-7)$ & $4(1-7)$ & $5(1-7)$ \\
\hline \multicolumn{4}{|c|}{$|S|^{b}(\min =0, \max =28)$ median (range) } \\
\hline Baseline & $8(0-24)$ & $11(0-27)$ & $10(0-21)$ \\
\hline 3 months follow-up & $7(0-20)$ & $11(0-27)$ & $9(0-27)$ \\
\hline 6 months follow-up & $6(0-18)$ & $13(0-25)$ & $9(0-18)$ \\
\hline \multicolumn{4}{|c|}{$\mathrm{FFMQ}^{\mathrm{b}}(\min =29, \max =145)$ mean $(\mathrm{SD})$} \\
\hline Baseline & $105.5(80-129)$ & $105.6(82-128)$ & $100.6(89-114)$ \\
\hline 3 months follow-up & $107.6(90-125)$ & $103.5(81-129)$ & $103.8(92-117)$ \\
\hline 6 months follow-up & $106.8(85-132)$ & $102.5(83-127)$ & $102.8(87-116)$ \\
\hline \multicolumn{4}{|c|}{$\mathrm{SRH}^{\mathrm{b}}(\min =1 \max =5)$ median (range) } \\
\hline Baseline & $3(1-4)$ & $3(2-5)$ & $3(2-5)$ \\
\hline 3 months follow-up & $3(1-4)$ & $3(2-5)$ & $4(2-5)$ \\
\hline 6 months follow-up & $4(1-4)$ & $4(2-5)$ & $4(2-5)$ \\
\hline
\end{tabular}

${ }^{a}$ Percentage of sedentary time and time in different intensity of physical activity per valid day and averaged over the number of valid days, activity monitor measured

${ }^{b}$ Self-reported measurements. Data are presented as mean values and standard deviation (SD) for normally distributed variables, and as median and range for variables with skewed distribution and variables based on nominal scales. LIPA light physical activity, MVPA moderate to vigorous physical activity, ISI insomnia severity index, FFMQ five facets of mindfulness questionnaire, SRH self-rated health

group comprised both PAP and mindfulness, meaning an individually adjusted PAP combined with an addition of the same mindfulness course as in the mindfulness group.

\section{Statistics}

\section{Power calculation}

Sample size of a full-scale intervention study with a follow-up time at 12 months was calculated on a 1:1 relationship between two groups (PAP and combination), and estimated to $n=375$ in each group, based on a power analysis with $5 \%$ significant-level and a power of $80 \%$. Drop out was expected to be $30 \%$. The calculation was based on other studies with self-reported compliance to PAP as an outcome measure, where $50 \%$ of the participants followed the recommendation on physical activity from the PAP. We estimated an increase from 
$50 \%$ to $62.5 \%$ of self-reported adherence to PAP [36]. The pilot-study sample size is based on the assumed patient flow and due to a limited project budget [37]. In this pilot study, we aimed to include approximately 30 participants in each arm, which is in concordance to a general flat rule, using a minimum of 30 participants to be able for estimating a parameter [38].

\section{Randomisation}

The randomisation to the three intervention groups (PAP, combination, mindfulness) was stratified by the patients' age and sex, with a total of three age groups: $40-49,50-59$ and $60-65$ years. The randomisation was done by a minimisation method with a random element, as minimisation variables in the randomisation we used age and sex to get the groups as equal as possible [39]. The randomisation was done in the statistical programme STATA version 15 (StataCorp, College Station, TX).

\section{Statistical methods}

The intervention effect on changes in outcome measures was examined by analysing average group differences (PAP, combination, mindfulness) in baseline score and change in each outcome between baseline and 3 and 6 months follow-up using a linear mixed-effects model. Each model included the time variable and group as indicator variables, and an interaction between time and group to estimate treatment differences in change over time, adjusted for baseline measures and taking the correlation between repeated measurements into account. We did not adjust for the minimisation variables in the analysis. Statistical analyses were done using STATA version 15 (StataCorp, College Station, TX).

\section{Results}

\section{Recruitment}

For the period 1st of September 2016 until the 31st of December 2018, a total of 136 eligible patients were asked to participate in the study and 88 were included. The median age among the participants in the PAPgroup was 54 years; in the combination-group, it was 54 years and in the mindfulness-group it was 53 years (Table $1)$. Among those who declined participation, the average age was 56 years ( 29 women and 19 men) (Fig. 1).

\section{Fulfilment of feasibility criteria}

We monitored several feasibility criteria to evaluate the suitability of the study design [27]. If all the feasibility criteria were fulfilled, the main study was considered possible to conduct without further changes in the protocol. If the criteria were not fulfilled, the protocol was considered to need adjustment, and if the criteria were fulfilled to less than $70 \%$ it was considered not possible to carry on with a full-scale study in the current form.

A recruitment rate of $30 \%$ was considered to be successful; 88 (64.7\%) of all the 136 patients eligible for the study, who were asked to participate, accepted.

A dropout rate of less than $30 \%$ was considered successful: during the study, there were a total of $20.4 \%$ (5 men, 13 women) dropouts with an average age of 52 .

A successful attendance rate to the mindfulness course was set to $\geq 70 \%$, of those randomised to any of the groups containing mindfulness should participate in at least $75 \%$ of the mindfulness meetings. There were $52 \%$ ( $n=15$ in the mindfulness group, $n=16$ in the combination) who attended six times or more to the meetings.

Seventy percent of patients randomised to any group containing mindfulness should practise mindfulness for at least $20 \mathrm{~min}$ with the web-based application at least 5 days a week (a total of $800 \mathrm{~min}$ or more). The mean time spent in web-based training, during the study, was $184.69 \mathrm{~min}$ with a standard deviation of $330.93 \mathrm{~min}$ (minimum 0 and max $1300 \mathrm{~min}$ ). Only 8\% (seven persons) did $800 \mathrm{~min}$ or more $(n=4$ in the mindfulness group, $n=3$ in the combination).

\section{Secondary outcome}

The patients were randomised into the three groups, PAP $(n=29)$, combination $(n=29)$, or mindfulness $(n=30)$. In the PAP-group, there were $24.1 \%$ (2 men, 5 women) dropouts with an average age of 53 years. In the mindfulness group, there were 20\% (3 men, 3 women) dropouts with an average age of 50 years. The combination group had $17.2 \%$ (5 women) of dropouts with an average age of 53 years (Fig. 1). Two dropouts were due to illness, five persons did not show up at follow-ups even after two reminders. Six individuals did not want to continue without giving any reason, four people cited lack of time and one person moved and could not continue participating in the study (Fig. 1). After exclusion of those with fewer than four valid activity monitor wear days, $n=$ 26 in the PAP-, $n=26$ in the combination- and $n=$ 25 in the mindfulness group remained. The wear time with activity monitors differed between 0 and 12 days. There were no significant baseline differences between the dropouts and the remaining participants (see Additional file 1). There were over $80 \%$ of the participants at each time-point who wore the accelerometer for 4 days or more (see Additional file 2)

In the sensitivity analyses, we analysed the data in several different ways, both with one valid activity monitor day (see Additional file 3), and four valid activity monitor days (Table 2), with similar results. 
CONSORT diagram over included patients from baseline to six month follow-up

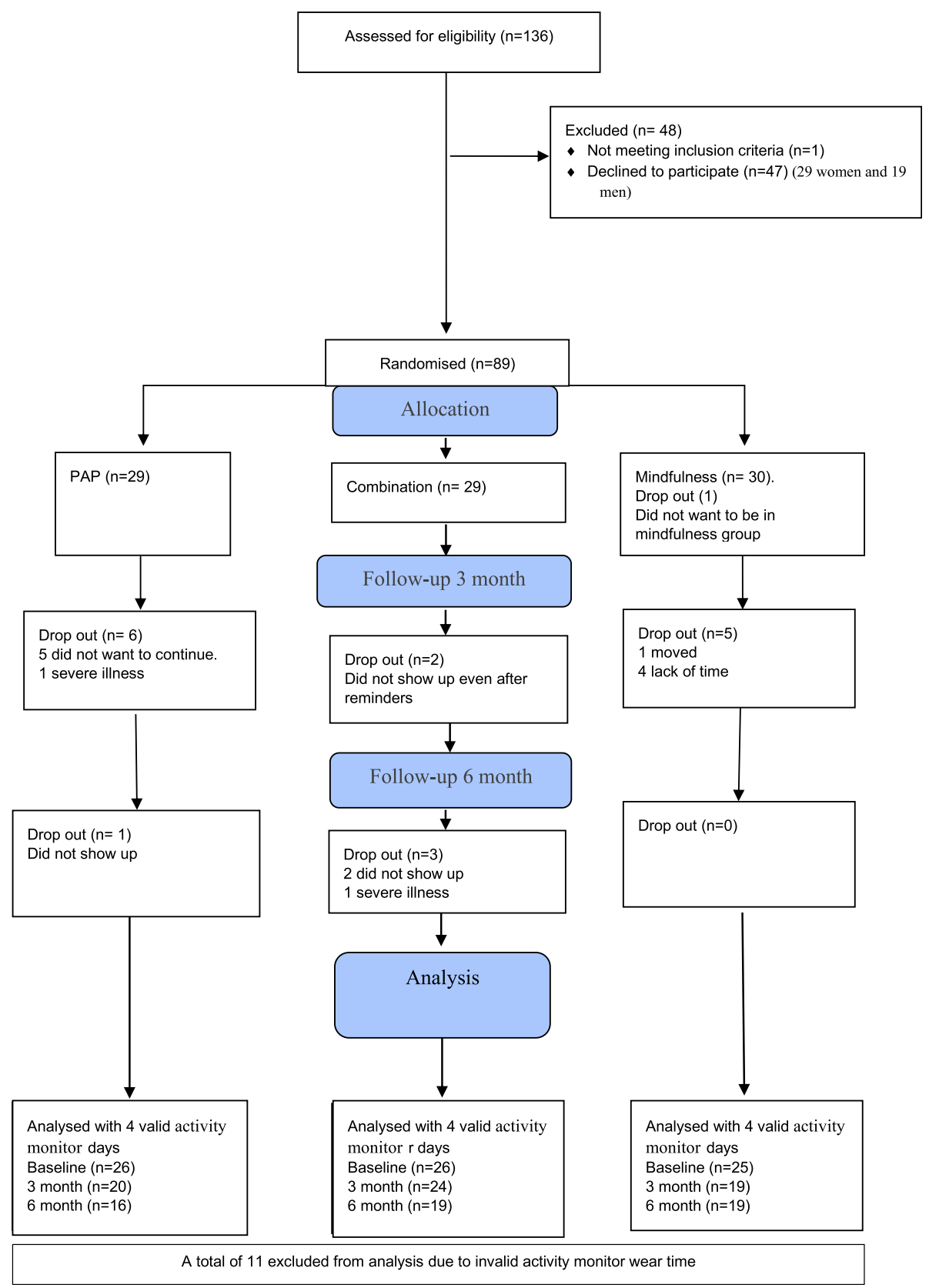

Fig. 1 CONSORT diagram over included patients from baseline to 6-month follow-up

\section{Intervention outcomes}

\section{Differences in change between intervention groups}

Regarding group differences in alteration over time, percentage sedentary time showed only small and nonsignificant indications of differences between the three groups (95\% CI - 1.74; 0.22; Table 2; Fig. 2). The same signals of suggested alteration were seen in differences regarding change in mean percentage of time in LIPA
(95\% CI - 0.35; 1.47; Table 2; Fig. 3) between the three groups. There was only a minor alteration in mean percentage of time in MVPA, and no differences in change between the three groups $(95 \% \mathrm{CI}-0.19 ; 0.48$; Table 2; Fig. 4).

Self-reported leisure time activity increased in all groups but did not show any overall difference in change (95\% CI - 0.03; 0.49) between the groups (Table 2; Fig. 5). The 
Table 2 Intercept (adjusted baseline value) and changes from baseline to 3 and 6 months in the three groups using mixed-effect models. Individuals with at least 4 valid days (600 min activity monitor wear time per day)

\begin{tabular}{|c|c|c|c|c|}
\hline Outcome & $\begin{array}{l}\text { Adjusted } \\
\text { baseline } \\
\text { value }\end{array}$ & $\begin{array}{l}\text { Change from } \\
\text { baseline to } 3 \text { months }\end{array}$ & $\begin{array}{l}\text { Change from } \\
\text { baseline to } 6 \text { months }\end{array}$ & $\begin{array}{l}\text { Overall mean difference } \\
\text { between groups over time } \\
(95 \% \mathrm{Cl})\end{array}$ \\
\hline \multicolumn{4}{|l|}{ Sedentary $^{a}$ (percentage) } & \multirow{4}{*}{$\begin{array}{l}-0.75 \\
(-1.74 ; 0.22)\end{array}$} \\
\hline PAP & 66.3 & 0.5 & 0.2 & \\
\hline Mindfulness & 65.8 & 1.1 & 1.4 & \\
\hline Combination & 66.4 & -0.2 & -2.8 & \\
\hline \multicolumn{4}{|l|}{ LIPA $^{a}$ (percentage) } & \multirow{4}{*}{$\begin{array}{l}0.56 \\
(-0.35 ; 1.47)\end{array}$} \\
\hline PAP & 30.9 & 0.01 & 0.03 & \\
\hline Mindfulness & 31.7 & -1.1 & -1.3 & \\
\hline Combination & 30.5 & -0.9 & 2.4 & \\
\hline \multicolumn{4}{|l|}{ MVPA $^{a}$ (percentage) } & \multirow{4}{*}{$\begin{array}{l}0.15 \\
(-0.19 ; 0.48)\end{array}$} \\
\hline PAP & 2.9 & -0.5 & -0.2 & \\
\hline Mindfulness & 2.5 & -0.1 & -0.1 & \\
\hline Combination & 3.1 & 1.0 & 0.2 & \\
\hline \multicolumn{4}{|l|}{ Leisure time activity $(1-6)^{b}$} & \multirow{4}{*}{$\begin{array}{l}0.23 \\
(-0.03 ; 0.49)\end{array}$} \\
\hline PAP & 1.67 & 1.11 & 1.18 & \\
\hline Mindfulness & 1.88 & -0.05 & 0.59 & \\
\hline Combination & 1.81 & 1.62 & 2.00 & \\
\hline \multicolumn{4}{|l|}{ Daily activity $(1-7)^{b}$} & \multirow{4}{*}{$\begin{array}{l}1.93 \\
(-0.22 ; 0.26)\end{array}$} \\
\hline PAP & 3.40 & 1.21 & 1.09 & \\
\hline Mindfulness & 3.63 & 0.39 & 0.77 & \\
\hline Combination & 3.42 & 1.16 & 1.14 & \\
\hline \multicolumn{4}{|l|}{ Weight (kg) } & \multirow{4}{*}{$\begin{array}{l}0.18 \\
(-0.32 ; 0.68)\end{array}$} \\
\hline PAP & 90.9 & -1.8 & -2.4 & \\
\hline Mindfulness & 85.3 & -0.13 & -0.6 & \\
\hline Combination & 84.8 & -0.74 & -1.6 & \\
\hline \multicolumn{4}{|l|}{$\mathrm{SRH}^{\mathrm{C}}(1-5)$} & \multirow{4}{*}{$\begin{array}{l}0.8 \\
(-0.06 ; 0.21)\end{array}$} \\
\hline PAP & 3.2 & 0.2 & 0.2 & \\
\hline Mindfulness & 3.3 & 0.3 & 0.3 & \\
\hline Combination & 3.3 & 0.4 & 0.5 & \\
\hline
\end{tabular}

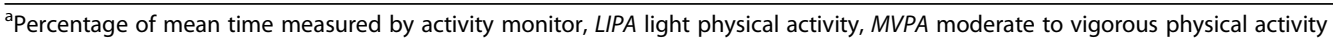

${ }^{b}$ Self-reported measurements: leisure time activity on a scale from $0=0 \mathrm{~min}$ per week, $6 \geq 120 \mathrm{~min} /$ week Daily activity on a scale $0=0$ min per week, $7 \geq 300$ min per week

'Self-rated health

${ }^{d}$ interaction between all three groups and timepoints

same pattern was seen in self-reported daily activity, with no difference in change between groups over time $(95 \%$ CI - 0.22; 0.26; Table 2; Fig. 6).

\section{Secondary intervention outcomes}

The analysis did not show any indications of large differences in change between groups regarding SRH (Table 2; Fig. 7), ISI (see Additional file 4) or FFMQ (see Additional file 4). The indicated alteration of blood pressure (see Additional file 4), weight (Table 2; figure see Additional file 5), BMI (see Additional file 4 for table, see Additional file 6 for figure) and blood lipids (see Additional file 4) did not suggest any statistically significant differences in change over time between the three groups.

\section{Discussion \\ Main findings of the study \\ Feasibility}

Since only two of the four feasibility criteria were fulfilled, major changes in the mindfulness intervention design should be considered before we can conduct a full study. Even if the drop-out rate was acceptable, according to the feasibility criteria, a sample size calculation to a full study must consider the loss to follow-up. 


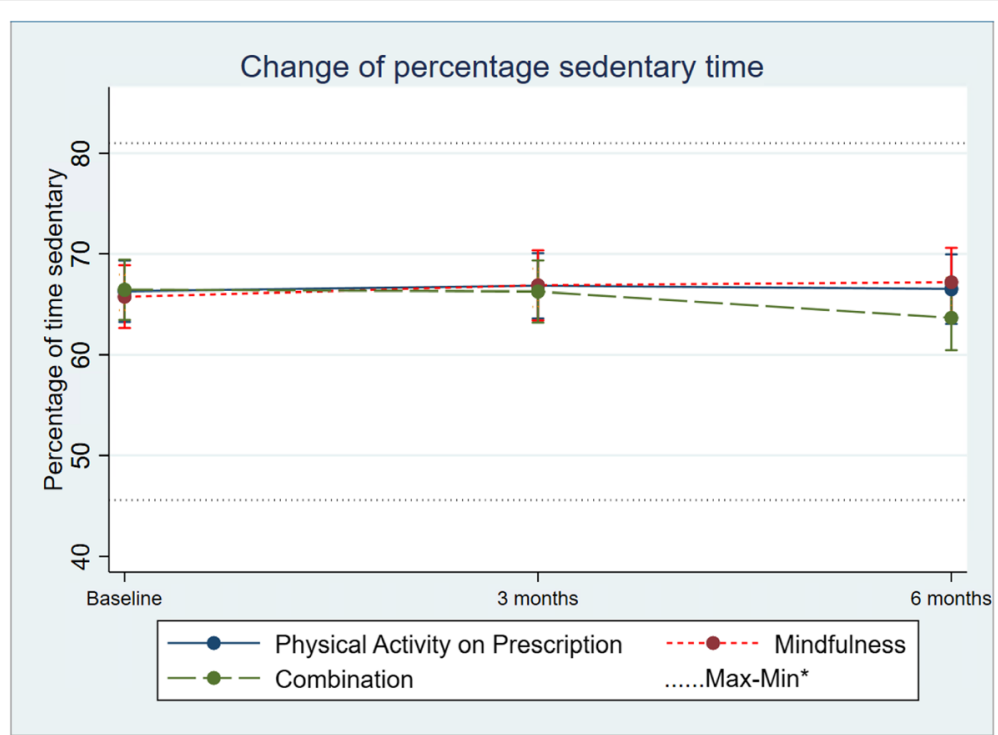

Fig. 2 Change in percentage sedentary in 1 the three groups over time. Differences between and within the groups are estimated by a mixedeffect model. *Maximum and minimum value of all observations

In the present pilot-study, there were 11 participants excluded from analysis due to low activity monitor wear time. In an attempt to increase the adherence to wear the activity monitor, participants should perhaps get the opportunity to choose the type of activity monitor, i.e. worn by the hip or by the wrist. However, using activity monitors measuring from different places of the body (wrist or hip) may affect the ability to conduct a correct analysis of the measurements. In a modified study, comparing the three groups (PAP, mindfulness and combination), we may need to adjust what time of day the mindfulness courses are arranged, and perhaps adjust the length of time for the daily exercise. Spending 20 min per day doing mindfulness may be hard to fit into one's ordinary schedule and even more difficult to combine with increased physical activity.

\section{Intervention outcome}

The main intervention outcome was to compare differences in change over time of physical activity level

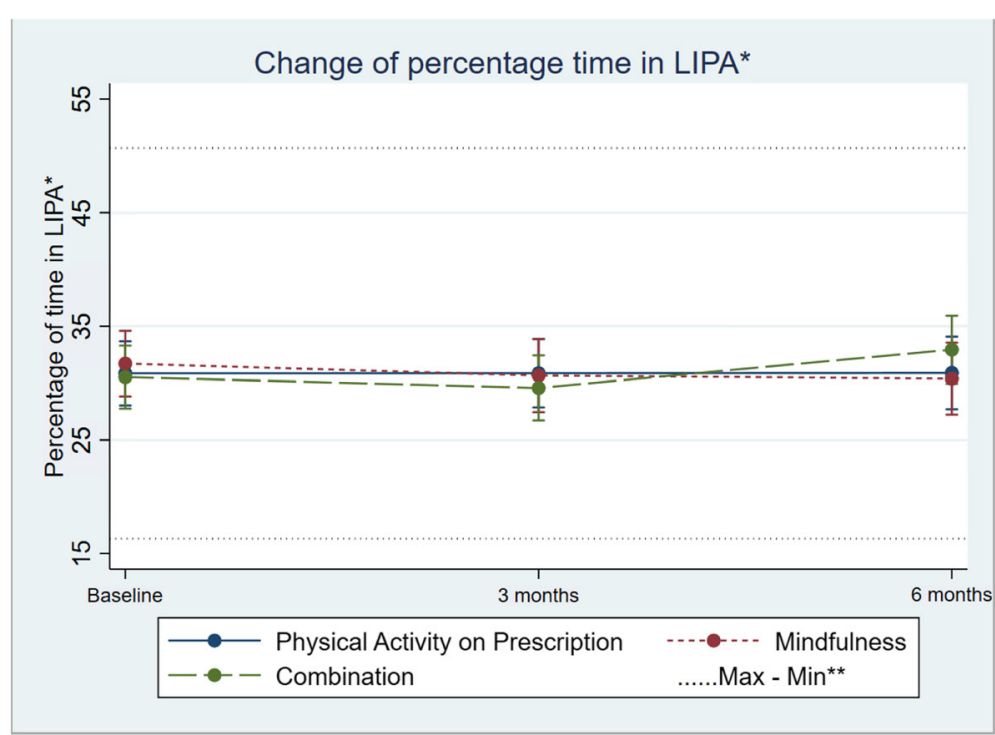

Fig. 3 Change in percentage light physical activity (LIPA), in the three groups over time. Differences between and within the groups are estimated by a mixed-effect model. **Maximum and minimum value of all observations 


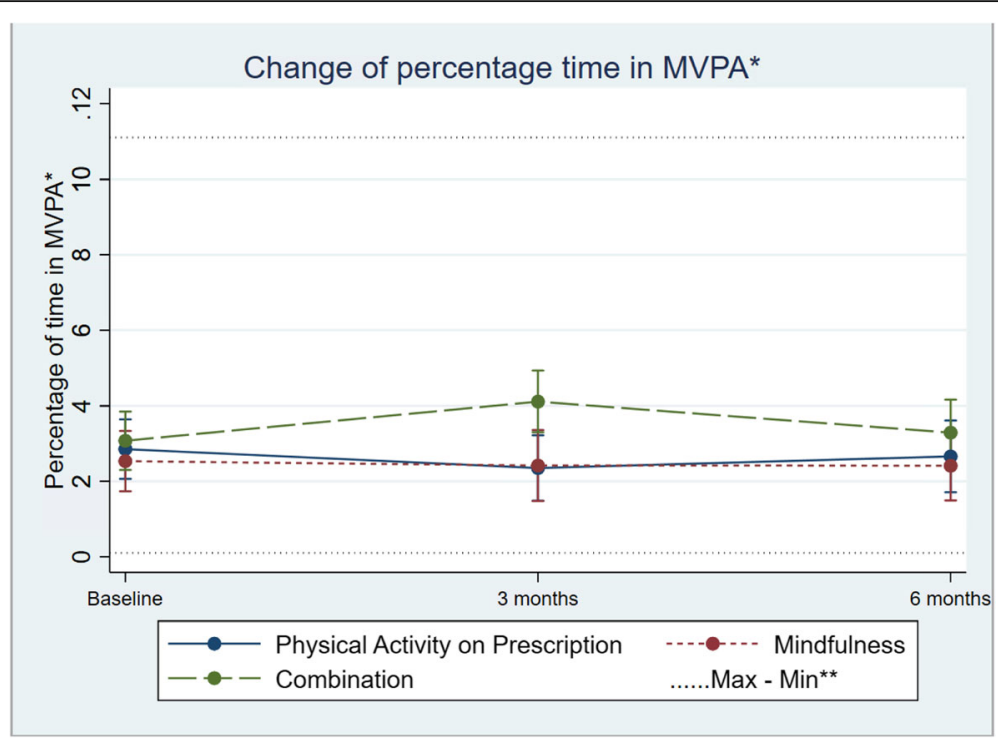

Fig. 4 Change in percentage moderate to vigorous physical activity (MVPA), in the three groups over time. Differences between and within the groups are estimated by a mixed-effect model. *Moderate to vigorus physical activity. ** Maximum and minimum value of all observations

between three groups: PAP, mindfulness and a combination containing both PAP and mindfulness. The results showed no differences between the groups regarding activity monitor measurements. Neither were there any differences in change of self-reported activity. We did not find any indications that mindfulness alone increased the percentage of time in physical activity, according to the data from the activity monitors. The combination seemed to increase LIPA, self-reported physical activity and SRH more than the other interventions in within-group comparisons, even if there was no difference in change between the groups.

Even if PAP and the combination seemed to increase self-reported physical activity more than mindfulness alone, the changes were small. The 2.4 percentage points increase of LIPA at 6 months compared with baseline in the combination group represented about 15 min more per day on average, based on a count with an activity monitor wear time of 600 min per day. Nevertheless, the small decrease in sedentary behaviour and increase in

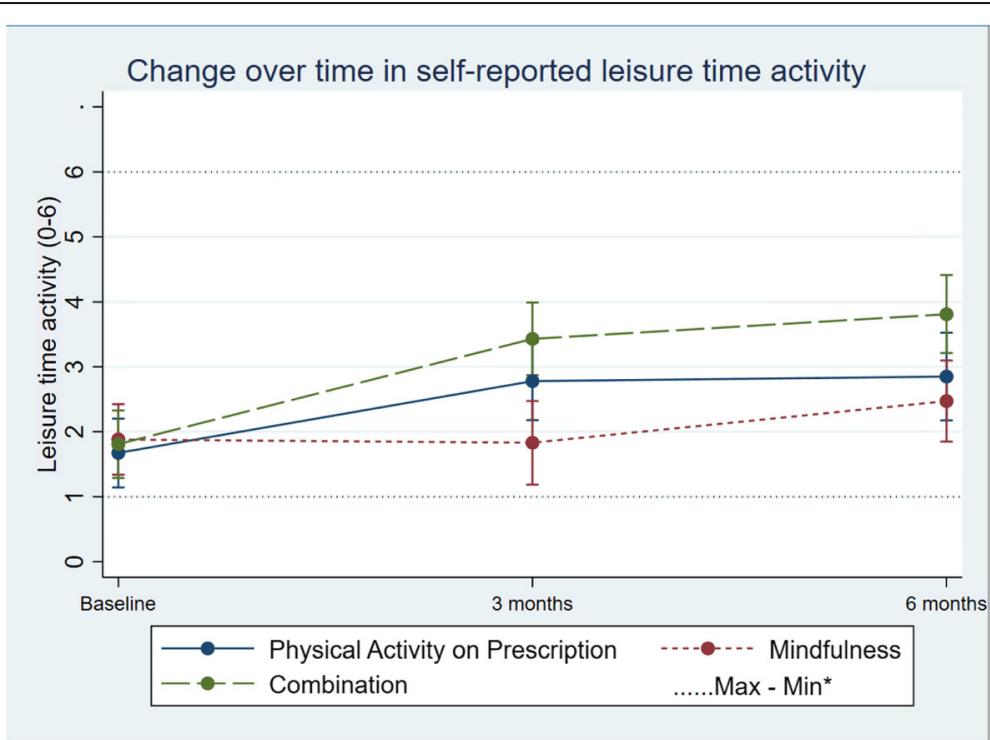

Fig. 5 Change in units of self-reported leisuretime activity in the three groups over time. Differences between and within the groups are estimated by a mixed-effect model. * Maximum and minimum value of all observations 


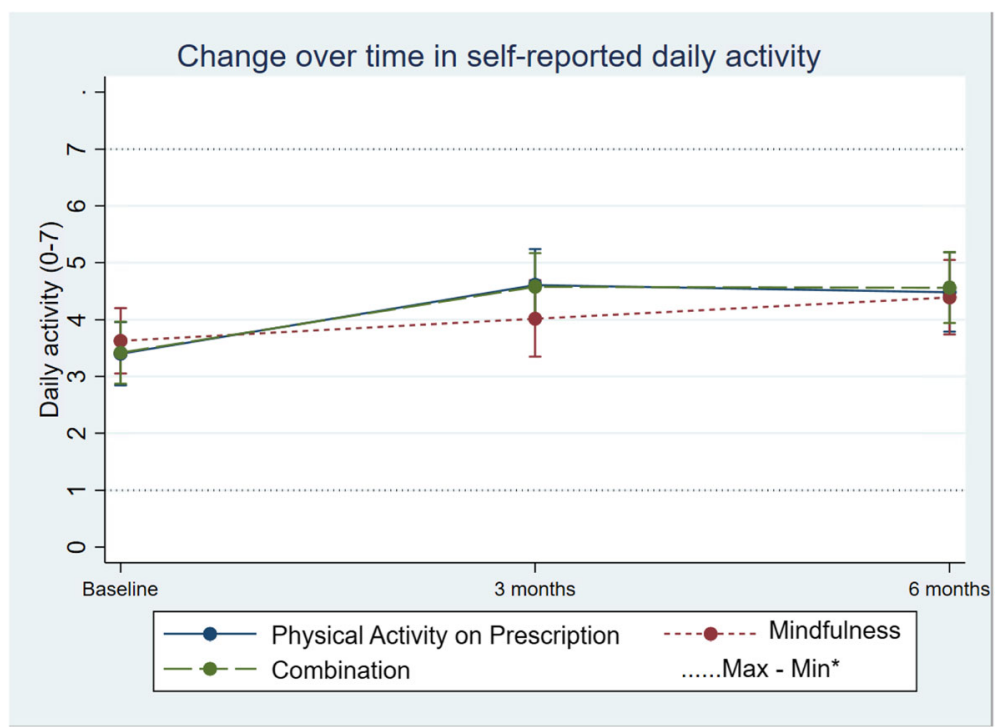

Fig. 6 Change in units of self reported daily activity in the three groups over time. Differences between and within the groups are estimated by a mixed-effect model. *Maximum and minimum value of all observations

physical activity during the short follow-up can be seen as a positive outcome. With a longer follow-up, we may have seen a bigger change, considering that it is a major life challenge to change from being mostly sedentary to being more active. On the other hand, previous research has indicated that the self-reported effect of PAP is most pronounced during the first 3 months [36].

The discrepancy between the activity monitoring and the self-reported activity seen in the present study may be explained by low physical activity in the week when measured with an activity monitor, and thus not representative for the physical activity in an average week for the patient. However, it is a known fact that the selfreported activity level increases more over time compared to objective measurements, especially with repeated measurements [31, 40]. PAP is, at present, in the Swedish healthcare system, the only accessible tool for motivating inactive people to increase their overall activity level. PAP has indeed shown effectiveness according to Onerup et al. [11], but the findings in our study could

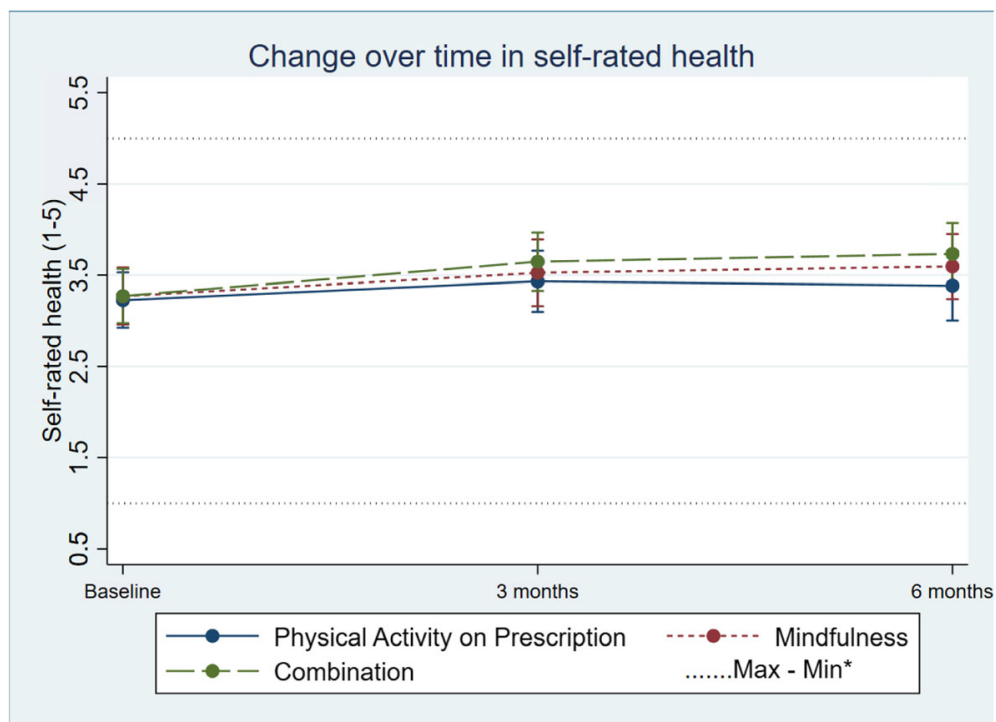

Fig. 7 Change in units of self-rated health in the three groups over time. Differences between and within the groups are estimated by a mixedeffect model. *Maximum and minimum value of all observations 
not confirm this effect with activity monitors. Our results are consistent with a previous study [12], which failed to detect any significantly increased MVPA among patients who received PAP. Since using PAP in healthcare is time-consuming, it is important to examine if the method is effective. Therefore, larger controlled trials with PAP and activity monitors are needed in order to evaluate the effect. To obtain the participants' true activity pattern, it is important to complete self-reported activity with activity monitors, perhaps over several weeks.

A noteworthy finding is the increase in units of SRH within all groups (Table 2; Fig. 7), which can depend on the same fact as other self-reported values that increase with repeated measurements [31, 40]. Both mindfulness $[18,41]$ and physical activity $[42,43]$ have been associated with increased levels of SRH; thus, this may be an explanation of the increased SHR in all groups.

We invited all patients, who reported themselves as physically inactive regardless of diagnosis, thus representing a usual cohort of patients in a Swedish primary health care clinic. It is possible that the results might have been different if we included a more specified group of patients. Hence, our results suggest that mindfulness may have a motivating effect. However, the small tendencies need to be confirmed by a larger study.

\section{Strengths}

This is one of the first randomised trials with the Swedish PAP model and mindfulness aiming at a broad primary health care population with objective measurements of physical activity. According to the baseline activity monitor data, we managed to capture the most sedentary patients with a low percentage of physical activity, which was the aim. The high recruitment rate and low dropout rate indicates that patients are interested in participating in these types of studies, and thus a marker for the possibility to obtain enough participants in a bigger study with the same aim as the present study.

\section{Limitations}

This pilot study is underpowered compared with the planned full study, which can be the reason that we failed to show significant differences between the groups regarding the activity monitor measured results. The limitation with the ACTi Graph GT1X activity monitor is that it only measures cardiorespiratory training and not other physical activities such as weightlifting, biking and swimming. Low compliance in wearing the activity monitor also compromised the reliability of the results (Table 2).

\section{Conclusions}

The study design needs adjustment for the mindfulness intervention design before a fully scaled study can be conducted. The combination of PAP and mindfulness may increase physical activity and SRH more than PAP or mindfulness alone.

\section{Abbreviations}

PAP: Physical activity on prescription; WHO: World Health Organization; ISI: Insomnia Severity Index; FFMQ: Five Facets of Mindfulness Questionnaire; LIPA: Light physical activity; MVPA: Moderate to vigorous physical activityMBSRMindfulness-Based Stress Reduction; MBCT: Mindfulness-Based Cognitive Therapy

\section{Supplementary Information}

The online version contains supplementary material available at https://doi. org/10.1186/s40814-021-00810-6.

Additional file 1. Table presenting differences in baseline values between dropouts and those who continued.

Additional file 2. Table presenting number of days with activity monitor wear time of 600 minutes or more per day.

Additional file 3. Intercept (adjusted baseline value) and changes from baseline to 3 and 6 months in the three groups using mixed effect models. Containing individuals with at least 1 valid day (600 minutes activity monitor wear time per day).

Additional file 4. Intercept (adjusted baseline value) and changes from baseline to 3 and 6 months in the three groups using mixed effect models. Containing analysis of BMI, total cholesterol, low-density cholesterol, high-density cholesterol, triglycerides, diastolic- and systolic blood pressure, insomnia severity scale and five facets of mindfulness questionnaire. Individuals with at least 4 valid days (600 minutes activity monitor wear time per day).

Additional file 5. Change in kilograms in the three groups over time. Differences between and within the groups are estimated by a mixed effect model.

Additional file 6. Change in BMI $\left(\mathrm{kg} / \mathrm{m}^{2}\right)$ in the three groups over time. Differences between and within the groups are estimated by a mixed effect model.

\section{Acknowledgements}

The authors wish to thank Ola Schenström and associates at Mindfulness centre for support regarding the mindfulness program. Mindfulness instructors, Lena Lind and Teresia Varga-Fridh, for leading the mindfulness groups, and all participants involved in the study. The authors also wish to thank the Center for Primary Healthcare Research science editor, Patrick Reilly, for his useful comments on the text.

\section{Authors' contributions}

P. N, study design, statistical analysis, writing and editing the manuscript. S.C, study design, writing and editing the manuscript. E.S, study design, writing, and editing the manuscript. K.P, statistical analysis, editing manuscript. E.E.H, study design, writing and editing the manuscript. K.S, study design, writing and editing the manuscript. J.S, writing and editing the manuscript. B.Z, study design, writing and editing the manuscript. All authors read and approved the final manuscript.

\section{Funding}

This work was supported by grants awarded to Dr. Bengt Zöller by the Swedish Heart-Lung Foundation, ALF funding from Region Skåne awarded to Dr. Bengt Zöller and grants awarded to Dr. Bengt Zöller by the Swedish Research Council. Grants awarded to Dr. Kristina Sundquist by the Swedish Heart-Lung Foundation. ALF funding from Region Skåne awarded to Susanna Calling. Open Access funding provided by Lund University. publicly available due to confidentiality for patients due to small study size but are available from the corresponding author. 


\section{Declarations}

\section{Ethics approval and consent to participate}

All parts of the study were conducted according to the principles of the 1964 Helsinki Declaration and the study was approved by the Regional Ethical Review Board in Lund (registration number 2016/404). The study is registered at ClinicalTrials.gov, registration number NCT02869854, registration date 17 August 2016, https://clinicaltrials.gov/ct2/show/NCT02869854. Written informed consent was obtained from all patients entering the study before inclusion.

\section{Consent for publication}

Not applicable

\section{Competing interests}

The authors declare that they have no competing interests.

\section{Author details}

'Center for Primary Health Care Research, Region Skåne, Lund, Sweden. ${ }^{2}$ Department of Clinical Sciences, Malmö Lund University, Lund, Sweden. ${ }^{3}$ Department of Health Sciences/Physiotherapy, Lund University, Lund, Sweden.

Received: 13 April 2020 Accepted: 2 March 2021

Published online: 17 March 2021

\section{References}

1. Yusuf S, Hawken S, Ounpuu S, Dans T, Avezum A, Lanas F, et al. Effect of potentially modifiable risk factors associated with myocardial infarction in 52 countries (the INTERHEART study): case-control study. Lancet. 2004; 364(9438):937-52.

2. Maher JP, Doerksen SE, Elavsky S, Hyde AL, Pincus AL, Ram N, et al. A daily analysis of physical activity and satisfaction with life in emerging adults. Health Psychol. 2013:32(6):647-56.

3. Penedo FJ, Dahn JR. Exercise and well-being: a review of mental and physical health benefits associated with physical activity. Curr Opin Psychiatry. 2005;18(2):189-93.

4. Zschucke E, Gaudlitz K, Strohle A. Exercise and physical activity in mental disorders: clinical and experimental evidence. J Prev Med Public Health. 2013;46(Suppl 1):S12-21

5. Gerovasili V, Agaku IT, Vardavas Cl, Filippidis FT. Levels of physical activity among adults 18-64 years old in 28 European countries. Prev Med. 2015;81: 87-91.

6. Loyen A, Van Hecke L, Verloigne M, Hendriksen I, Lakerveld J, SteeneJohannessen J, et al. Variation in population levels of physical activity in European adults according to cross-European studies: a systematic literature review within DEDIPAC. Int J Behav Nutr Phys Act. 2016:13:72

7. Ekblom-Bak E, Olsson G, Ekblom O, Ekblom B, Bergstrom G, Borjesson M. The Daily Movement Pattern and Fulfilment of Physical Activity Recommendations in Swedish Middle-Aged Adults: The SCAPIS Pilot Study. PLoS One. 2015;10(5):e0126336.

8. Orrow G, Kinmonth AL, Sanderson S, Sutton S. Effectiveness of physical activity promotion based in primary care: systematic review and metaanalysis of randomised controlled trials. BMJ. 2012;344:e1389.

9. Leijon ME, Bendtsen P, Nilsen P, Festin K, Stahle A. Does a physical activity referral scheme improve the physical activity among routine primary health care patients? Scand J Med Sci Sports. 2009;19(5):627-36.

10. Morgan O. Approaches to increase physical activity: reviewing the evidence for exercise-referral schemes. Public Health. 2005;1 19(5):361-70.

11. Onerup A, Arvidsson D, Blomqvist A, Daxberg EL, Jivegard L, Jonsdottir IH, et al. Physical activity on prescription in accordance with the Swedish model increases physical activity: a systematic review. Br J Sports Med. 2019; 53(6):383-8. https://doi.org/10.1136/bjsports-2018-099598. Epub 2018 Nov 9.

12. Moren C, Welmer AK, Hagstromer M, Karlsson E, Sommerfeld DK. The Effects of "Physical Activity on Prescription" in Persons With Transient Ischemic Attack: A Randomized Controlled Study. J Neurol Phys Ther. 2016:40(3):176-83

13. Williams DM, Lewis BA, Dunsiger S, Whiteley JA, Papandonatos GD, Napolitano MA, et al. Comparing psychosocial predictors of physical activity adoption and maintenance. Ann Behav Med. 2008;36(2):186-94.
14. Hertel AW, Finch EA, Kelly KM, King C, Lando H, Linde JA, et al. The impact of expectations and satisfaction on the initiation and maintenance of smoking cessation: an experimental test. Health Psychol. 2008;27(3 Suppl): S197-206.

15. Baldwin AS, Rothman AJ, Jeffery RW. Satisfaction with weight loss: examining the longitudinal covariation between people's weight-lossrelated outcomes and experiences and their satisfaction. Ann Behav Med. 2009;38(3):213-24.

16. Baldwin AS, Baldwin SA, Loehr VG, Kangas JL, Frierson GM. Elucidating satisfaction with physical activity: an examination of the day-to-day associations between experiences with physical activity and satisfaction during physical activity initiation. Psychol Health. 2013:28(12):1424-41.

17. Tsafou KE, De Ridder DT, van Ee R, Lacroix JP. Mindfulness and satisfaction in physical activity: A cross-sectional study in the Dutch population. J Health Psychol. 2016;21(9):1817-27.

18. Brown KW, Ryan RM. The benefits of being present: mindfulness and its role in psychological well-being. J Pers Soc Psychol. 2003;84(4):822-48.

19. Kabat-Zinn J. Wherever you go, there you are : mindfulness meditation for everyday life. London: Piatkus; 1994. p. xix. 1, 281

20. Bishop SR, Lau M, Shapiro S, Carlson L, Anderson ND, Carmody J, et al. Mindfulness: A proposed operational definition. Clin Psychol Sci Pract. 2004; 11(3):230-41.

21. Ruffault A, Bernier M, Juge N, Fournier JF. Mindfulness May Moderate the Relationship Between Intrinsic Motivation and Physical Activity: A CrossSectional Study. Mindfulness. 2016;7(2):445-52.

22. Zeidan F, Baumgartner JN, Coghill RC. The neural mechanisms of mindfulness-based pain relief: a functional magnetic resonance imagingbased review and primer. Pain Rep. 2019;4(4):e759.

23. Mohammed WA, Pappous A, Sharma D. Effect of Mindfulness Based Stress Reduction (MBSR) in Increasing Pain Tolerance and Improving the Mental Health of Injured Athletes. Front Psychol. 2018;9:722.

24. Meyer JD, Torres ER, Grabow ML, Zgierska AE, Teng HY, Coe CL, et al. Benefits of 8-wk Mindfulness-based Stress Reduction or Aerobic Training on Seasonal Declines in Physical Activity. Med Sci Sports Exerc. 2018;50(9): $1850-8$.

25. Schneider J, Malinowski $P$, Watson PM, Lattimore $P$. The role of mindfulness in physical activity: a systematic review. Obes Rev. 2019;20(3):448-63.

26. Mothes H, Klaperski S, Seelig H, Schmidt S, Fuchs R. Regular aerobic exercise increases dispositional mindfulness in men: a randomized controlled trial. Mental Health Phys Activity. 2014;7:111-9.

27. Nymberg P, Ekvall Hansson E, Stenman E, Calling S, Sundquist K, Sundquist J, et al. Pilot study on increased adherence to physical activity on prescription (PAP) through mindfulness: study protocol. Trials. 2018;19(1):563.

28. King AC, Haskell WL, Taylor CB, Kraemer HC, DeBusk RF. Group- vs homebased exercise training in healthy older men and women. A communitybased clinical trial. Jama. 1991;266(11):1535-42.

29. Sundquist J, Lilja A, Palmer K, Memon AA, Wang X, Johansson LM, et al. Mindfulness group therapy in primary care patients with depression, anxiety and stress and adjustment disorders: randomised controlled trial. $\mathrm{Br}$ J Psychiatry. 2015;206(2):128-35.

30. Gluck TM, Maercker A. A randomized controlled pilot study of a brief webbased mindfulness training. BMC psychiatry. 2011;11:175.

31. Troiano RP, Berrigan D, Dodd KW, Masse LC, Tilert T, McDowell M. Physical activity in the United States measured by accelerometer. Med Sci Sports Exerc. 2008:40(1):181-8.

32. Matthews $C E$, Chen KY, Freedson PS, Buchowski MS, Beech BM, Pate RR, et al. Amount of time spent in sedentary behaviors in the United States, 2003-2004. Am J Epidemiol. 2008;167(7):875-81.

33. Dragioti E, Wiklund T, Alföldi P, Gerdle B. The Swedish version of the Insomnia Severity Index: Factor structure analysis and psychometric properties in chronic pain patients. Scand J Pain. 2016;9:22-7.

34. Lilja JL, Frodi-Lundgren A, Hanse JJ, Josefsson T, Lundh LG, Skold C, et al. Five Facets Mindfulness Questionnaire--reliability and factor structure: a Swedish version. Cogn Behav Ther. 2011;40(4):291-303.

35. folkhälsoinstitut S. FAR ${ }^{\oplus:}$ individanpassad skriftlig ordination av fysisk aktivitet. Öatersund: Statens folkhälsoinstitut: 2011.

36. Leijon ME, Bendtsen P, Stahle A, Ekberg K, Festin K, Nilsen P. Factors associated with patients self-reported adherence to prescribed physical activity in routine primary health care. BMC Fam Pract. 2010;11:38. 
37. Leon AC, Davis LL, Kraemer HC. The role and interpretation of pilot studies in clinical research. J Psychiatr Res. 2011;45(5):626-9.

38. Browne RH. On the use of a pilot sample for sample size determination. Stat Med. 1995;14(17):1933-40.

39. Pocock SJ, Simon R. Sequential treatment assignment with balancing for prognostic factors in the controlled clinical trial. Biometrics. 1975;31(1):103-15.

40. Forsen L, Loland NW, Vuillemin A, Chinapaw MJ, van Poppel MN, Mokkink LB, et al. Self-administered physical activity questionnaires for the elderly: a systematic review of measurement properties. Sports Med. 2010;40(7):601-23.

41. Auty KM, Cope A, Liebling A. A systematic review and meta-analysis of yoga and mindfulness meditation in prison: effects on psychological well-being and behavioural functioning. Int J Offender Ther Comp Criminol. 2017;61(6): 689-710.

42. Jepsen R, Dogisso TW, Dysvik E, Andersen JR, Natvig GK. A cross-sectional study of self-reported general health, lifestyle factors, and disease: the Hordaland Health Study. PeerJ. 2014;2:e609.

43. Sodergren M, Sundquist J, Johansson SE, Sundquist K. Physical activity, exercise and self-rated health: a population-based study from Sweden. BMC Public Health. 2008;8:352

\section{Publisher's Note}

Springer Nature remains neutral with regard to jurisdictional claims in published maps and institutional affiliations.

Ready to submit your research? Choose BMC and benefit from:

- fast, convenient online submission

- thorough peer review by experienced researchers in your field

- rapid publication on acceptance

- support for research data, including large and complex data types

- gold Open Access which fosters wider collaboration and increased citations

- maximum visibility for your research: over $100 \mathrm{M}$ website views per year

At BMC, research is always in progress.

Learn more biomedcentral.com/submissions 